\title{
Comportamento biomecânico do sistema prótese/implante em região anterior de maxila: análise pelo método de ciclagem mecânica
}

\author{
Mechanical behavior of prostheses / implant system in the anterior region of maxilla: \\ analysis by the method of cycling mechanics
}

Henrique Eiji TABUSE ${ }^{a}$, Cássia Bellotto CORRÊa ${ }^{a}$ Luís Geraldo VAZ

${ }^{a}$ Faculdade de Odontologia, UNESP - Univ Estadual Paulista, Araraquara, SP, Brasil

\begin{abstract}
Resumo
Introdução: $\mathrm{O}$ adequado posicionamento tridimensional dos implantes é indispensável para garantir a previsibilidade no tratamento com implantes dentários. Objetivo: Analisar comparativamente o comportamento mecânico do sistema prótese/implante em região anterior de maxila, diferindo os sistemas de encaixe e posicionamento dos implantes. Material e método: Utilizando um modelo prototipado de maxila, as situações estudadas foram: Grupo IC - implantes nos incisivos centrais e cantilever nos incisivos laterais; Grupo IL - implantes nos incisivos laterais e pônticos nos incisivos centrais; Grupo ICIL - implantes no incisivo central e no incisivo lateral, intercalados com elementos suspensos. Para cada situação estudada, foram utilizadas as três conexões protéticas: hexágono externo, hexágono interno e cone-Morse. O ensaio de ciclagem mecânica foi realizado com a aplicação de $100 \mathrm{~N}$ de carga e frequência de $15 \mathrm{~Hz}$ no cíngulo dos incisivos a $45^{\circ}$ com o longo eixo do dente, para simular o movimento mastigatório. Resultado: No ensaio de ciclagem mecânica, todos os modelos de todos os grupos com os três tipos de conexões protéticas atingiram um milhão de ciclos sem que ocorresse ruptura do parafuso, do componente protético ou da estrutura metálica. Conclusão: Com a metodologia e as condições empregadas, pode-se concluir que o comportamento mecânico das reabilitações implantossuportadas foi semelhante para os diferentes posicionamentos dos implantes e diferentes conexões protéticas.
\end{abstract}

Descritores: Implantação dentária; prótese dentária fixada por implante; estresse mecânico.

\begin{abstract}
Introduction: The adequate three-dimensional positioning of implants is essential to ensure predictability in dental implant treatment. Purpose: Was to comparatively evaluate the mechanical behavior of the system prosthesis/ implant in the anterior maxilla, differing the prosthetic connection and the placement of implants. Material and method: By a maxilla prototyped model the situations were studied: Group IC- implants in the central incisor positions and cantilevers in the lateral incisor positions; Group IL - implants in the lateral incisor positions and pontics in the central incisor positions; Group ICIL one implant in a lateral and one in a central incisor position, with a pontic and a cantilever in the other positions. For each situations were used the prosthetic connections, external hexagon, internal hexagon and Morse-taper. In fatigue test was applied load $100 \mathrm{~N}$ of frequency $15 \mathrm{~Hz}$ in the incisors cingulum $45^{\circ}$ to the long axis of the tooth to simulate a masticatory movement. Result: After the fatigue test all models of all groups with all three types of connections prosthetic reached 1 million cycles without rupture occurred screw, prosthetic component or metallic structure. Conclusion: According to methodology and conditions studied, can be concluded that the mechanical behavior of the implant-supported rehabilitation was similar to the different positions of the dental implants and the different prosthetic connections.
\end{abstract}

Descriptors: Dental implantation; dental prosthesis; stress, mechanical.

\section{INTRODUÇÃO}

Atualmente, a reabilitação oral com implantes osseointegrados apresenta índices de sucesso bastante elevados. Segundo o protocolo estabelecido por Branemark, com realização do procedimento cirúrgico em duas etapas, pôde-se verificar uma taxa de sucesso entre $95 \%$ e $99 \%$ durante um período de dez anos $^{1}$.
Entretanto, o comportamento biomecânico dos implantes dentais é diferente dos dentes naturais, devido ao fato de não possuírem ligamento periodontal; dessa forma, transmitem as cargas mastigatórias diretamente ao osso suporte ${ }^{2}$.

Essa característica de distribuição das cargas diretamente ao tecido ósseo tem como consequência a modificação dos tecidos 
adjacentes ao implante; dentre estes, o tecido ósseo, que sofre uma modificação para o estabelecimento de novas distâncias biológicas, da qual faz parte a reabsorção óssea. Se cargas excessivas forem aplicadas no implante osseointegrado, essa reabsorção óssea será acentuada ${ }^{3}$.

Estudos têm confirmado essa correlação positiva entre sobrecarga oclusal e reabsorção óssea em implantes osseointegrados $^{4,5}$. Assim, todo esforço deve ser feito para compreender a distribuição das cargas no sistema prótese/implante e tecido ósseo para, dessa forma, possibilitar o estabelecimento de um adequado posicionamento tridimensional do implante, evitando o carregamento excessivo e o insucesso do tratamento.

O posicionamento tridimensional do implante está também intimamente ligado ao sucesso do resultado estético das reabilitações implantossuportadas ${ }^{6}$. Dentre os critérios considerados para o sucesso de reabilitações com implantes dentais, está o estabelecimento de tecidos moles com adequado contorno, o perfil gengival e a presença de papilas interproximais ${ }^{7}$.

A região dos dentes anteriores superiores é uma região de grande atenção no momento da reabilitação, devido ao elevado comprometimento estético. Esta região, muitas vezes, é bastante vulnerável ao acometimento traumático, em que se podem perder estes elementos dentais de forma irreversível.

Em uma região desdentada anterior de quatro dentes, para colocação de um implante para cada elemento perdido, respeitando as distâncias biológicas já estabelecidas na literatura ${ }^{8-11}$, a distância intercaninos necessária seria de $29 \mathrm{~mm}$, sendo o diâmetro de cada implante de aproximadamente $4 \mathrm{~mm}$, a distância a ser respeitada entre implantes de $3 \mathrm{~mm}$ e, entre dente e implante, de $2 \mathrm{~mm}$. No entanto, grande parte dos pacientes não possui esse espaço disponível para respeitar o posicionamento tridimensional adequado.

Assim, é indicada a colocação de apenas dois ou três implantes associados a um ou dois elementos suspensos para a otimização do espaço e o ganho estético, como sugerem Tarnow et al. ${ }^{8}$. Com a necessidade da utilização de pônticos em prótese múltipla, os implantes de fixação estarão sujeitos a uma maior sobrecarga durante a mastigação.

Portanto, o conhecimento sobre biomecânica do sistema prótese/implante e o tecido ósseo é de extrema importância para assegurar o sucesso no tratamento com implantes dentais.

\section{PROPOSIÇÃO}

Este estudo tem o objetivo de analisar o comportamento mecânico da reabilitação de uma região anterior de maxila com quatro elementos dentários perdidos e apenas dois implantes, variando o posicionamento desses implantes e o tipo de conexão protética.

\section{MATERIAL E MÉTODO}

Por meio de prototipagem, foi confeccionado um modelo 3D da região anterior de maxila com distância intercaninos de $27,3 \mathrm{~mm}$, baseado em um banco de tomografias computadorizadas disponibilizado pelo Centro de Tecnologia e Inovação Renato Archer (CTI). Esse modelo de maxila anatômica foi gerado a partir de uma média das estruturas anatômicas da região (Figura 1).

O modelo prototipado foi copiado pela técnica de moldagem única com silicona de condensação leve e pesada (Zetaplus, Oranwash e Endurente - Zermack ${ }^{\circledR}$ - Roma, Itália), como mostra a Figura 2. O molde foi vazado com gesso pedra de baixa expansão de presa (Gesso-RIO tipo II, São Paulo, Brasil), aguardou-se a presa e o modelo foi então perfurado seguindo-se a sequência de fresas recomendadas pelo fabricante (Neodent Ltda, Curitiba, Brasil), com o auxílio da guia cirúrgica também prototipada (Figura 2). Uma peça reta (Kavo, Santa Catariana, Brasil) acoplada a um motor (Driller BLM600- Driller ${ }^{\circledR}$, São Paulo, Brasil) foi utilizada para fresagem dos modelos. Para a perfuração dos modelos, utilizou-se o Kit Cirúrgico com as brocas sequenciais sugeridas pelo fabricante do implante a ser instalado (Neodent Ltda).

Após a instalação dos implantes, uma nova moldagem de transferência dos implantes foi realizada. Essa moldagem de transferência dos implantes permite o vazamento da resina epóxi Araldite (Maxepoxi Industrial e Comercial Ltda, São Paulo, Brazil), com os implantes já em posição (Figura 3), possibilitando que as espiras do implante fiquem em íntimo contato com a resina, simulando o processo de osseointegração.

Três diferentes posicionamentos dos implantes foram estudados:

IC- Implantes cônicos na posição dos incisivos centrais e cantilever na região dos incisivos laterais, utilizando as conexões hexágono externo, hexágono interno e cone-Morse.

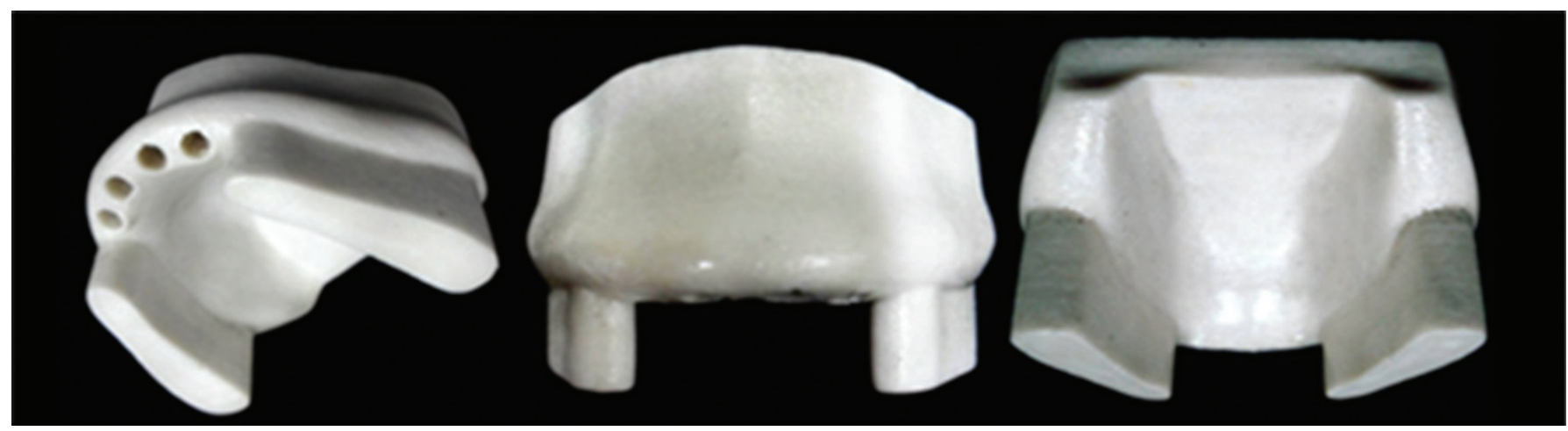

Figura 1. Modelo de maxila prototipado a partir de médias de tomografias computadorizadas, Centro de tecnologia e Inovação Renato Archer (CTI). 


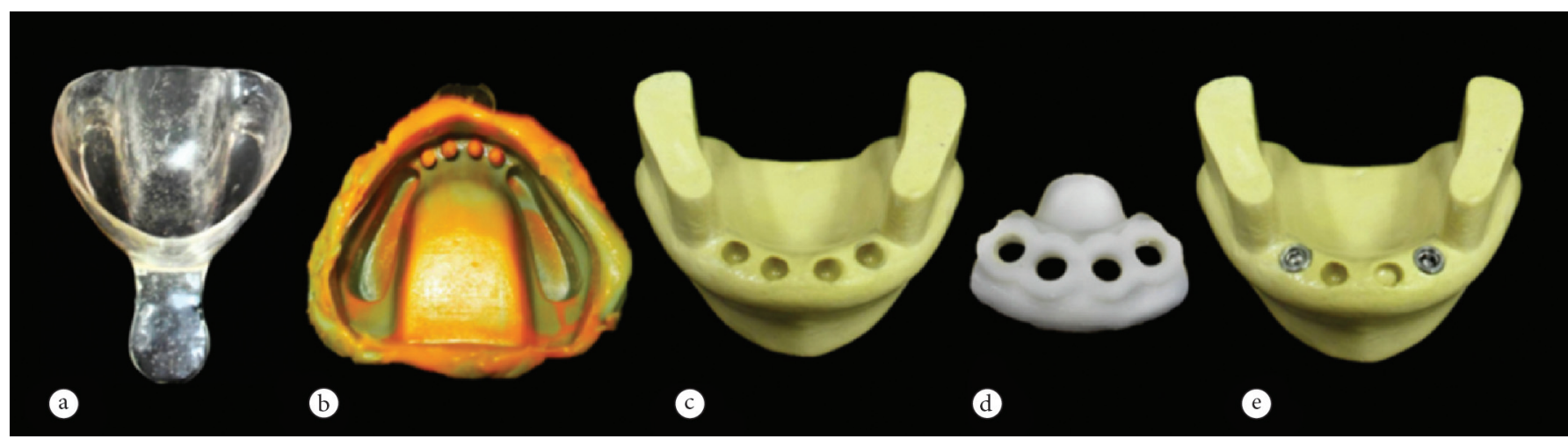

Figura 2. a-Moldeira Individual; b-Moldagem com silicona; c-Modelo duplicado em gesso; d-Guia cirúrgico; e-Modelo de gesso com os implantes.

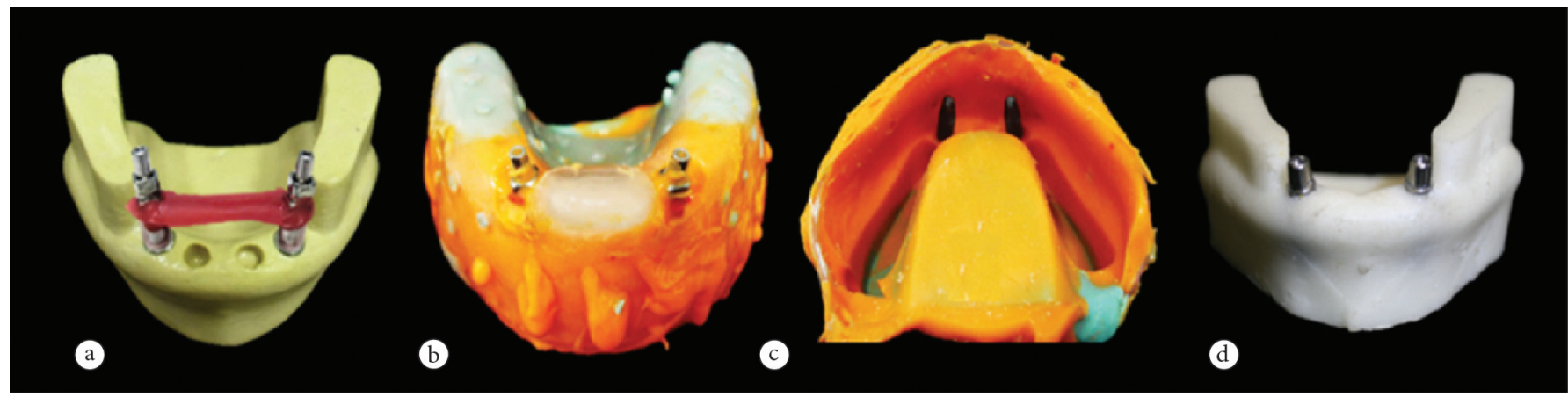

Figura 3. a-Transferentes; b-Moldagem; c-Implantes posicionados; d-Modelo em Resina Epóxi.
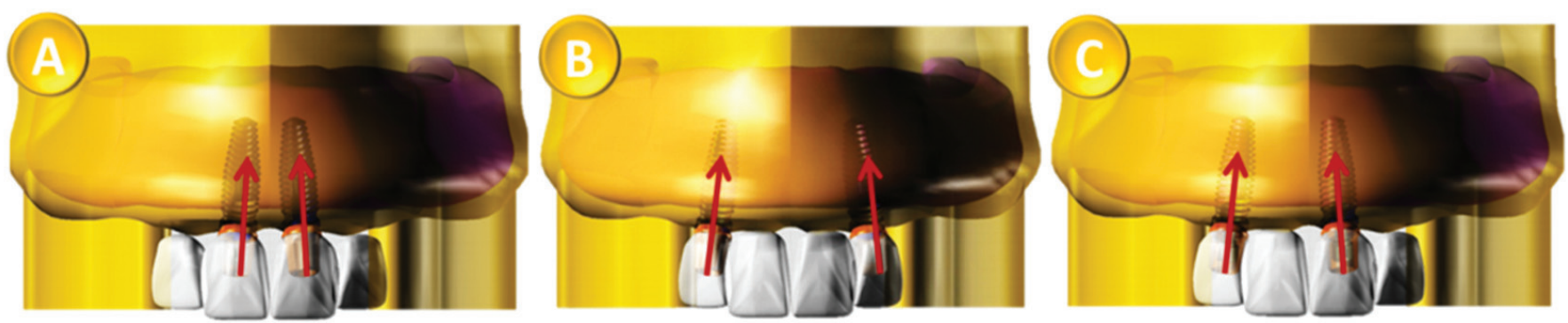

Figura 4. As três situações estudadas quanto à disposição dos implantes. Para cada situação (A, B e C), haverá três condições nas quais se alternará o tipo de conexão protética (hexágono interno, hexágono externo e cone-Morse).

IL- Implantes cônicos na posição dos incisivos laterais e pônticos na região dos incisivos centrais, utilizando as conexões hexágono externo, hexágono interno e cone-Morse

ICIL- Implantes cônicos alternados com pônticos e cantilever, utilizando as conexões hexágono externo, hexágono interno e cone-Morse (Figura 4).

Para duplicar os modelos de um mesmo grupo e variar o tipo de conexão protética utilizada, realizou-se a moldagem com o transferente do componente (munhão universal) (Figura 5). Padronizou-se, deste modo, o mesmo posicionamento dos implantes com os diferentes sistemas de encaixe (hexágono externo, hexágono interno e cone-Morse). Foram confeccionados cinco modelos para cada conexão protética, totalizando 15 modelos para cada grupo (IC, IL, ICIL).

A estrutura protética das três situações estudadas também foi prototipada (Figura 6). A duplicação das estruturas foi realizada pela moldagem com silicona leve e densa dos dentes prototipados posicionados no modelo (Figura 6). No molde, foi colocada resina Duralay (Polidental Indústria e Comércio Ltda, Cotia-SP,
Brasil). O molde com DuraLey foi reposicionado no modelo que continha os cilindros calcináveis posicionados nos componentes; após a presa da resina, o molde foi removido, obtendo-se uma cópia da estrutura prototipada. Um acabamento foi realizado nas peças em DuraLey e procedeu-se à fundição em liga $\mathrm{NiCr}$ (Verabond II) (Figura 6). A estrutura protética foi composta apenas pela estrutura metálica sem recobrimento estético.

\section{Ensaio de Ciclagem mecânica:}

Segundo Cetlin, Silva ${ }^{12}$, geralmente os processos de fadiga podem ser divididos em três estágios distintos:

Estágio 1 - Estágio inicial de deformação plástica, em que a nucleação da trinca é gerada devido a forças de cisalhamento, não sendo possível a sua observação a olho nu.

Estágio 2 - Fratura característica do processo de fadiga. Nesse estágio, há a propagação da trinca perpendicular ao sentido da tensão principal de tração, sendo sempre visível a olho nu.

Estágio 3 - Estágio em que a trinca do material se encontra na sua dimensão mais crítica, resultando em sua fratura final. Esta 
fadiga desenvolve-se progressivamente, dependendo, portanto, da tensão e das características mecânicas do material.

Por meio da ciclagem mecânica, o implante dental pode desenvolver microtrincas internas, as quais podem aumentar em número e tamanho conforme a quantidade de ciclos. Em um conjunto implante/componente protético, a ciclagem mecânica pode levar a micromovimentos neste sistema, podendo resultar em afrouxamento do conjunto, fendas no implante, trincas no parafuso de união do componente protético ou até mesmo a ruptura do implante dental, do componente ou do parafuso protético $^{13}$.
Ensaios de ciclagem mecânica para elementos unitários em região anterior já são descritos na literatura, segundo Merz et al. ${ }^{14}$, Huang et al. ${ }^{15}$, Tsuge, Higawara ${ }^{16}$ e Corrêa et al. ${ }^{17}$. Entretanto, neste estudo, devido à complexidade dos modelos - quatro elementos inter-relacionados de uma estrutura protética em região anterior de maxila -, um novo dispositivo capaz de simular a condição de angulação vertical e angulação horizontal das forças incidentes nessa região foi desenvolvido (Figura 7). O dispositivo desenvolvido foi composto de uma barra cilíndrica acoplada em um cone que, ao movimentar-se para baixo, empurrava hastes cilíndricas e estas, por sua vez, aplicavam

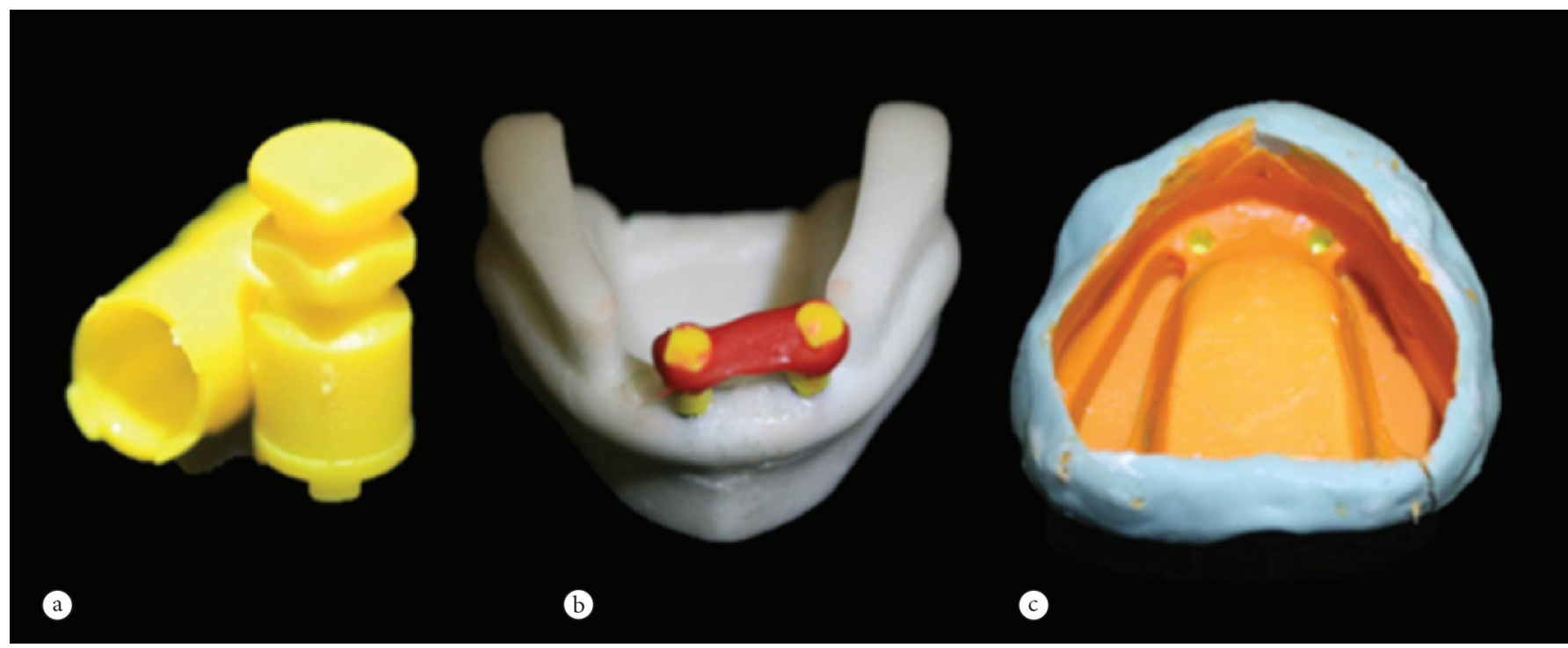

Figura 5. a-Transferente do munhão; b-União dos transferentes; c-Molde com o transferente em posição.

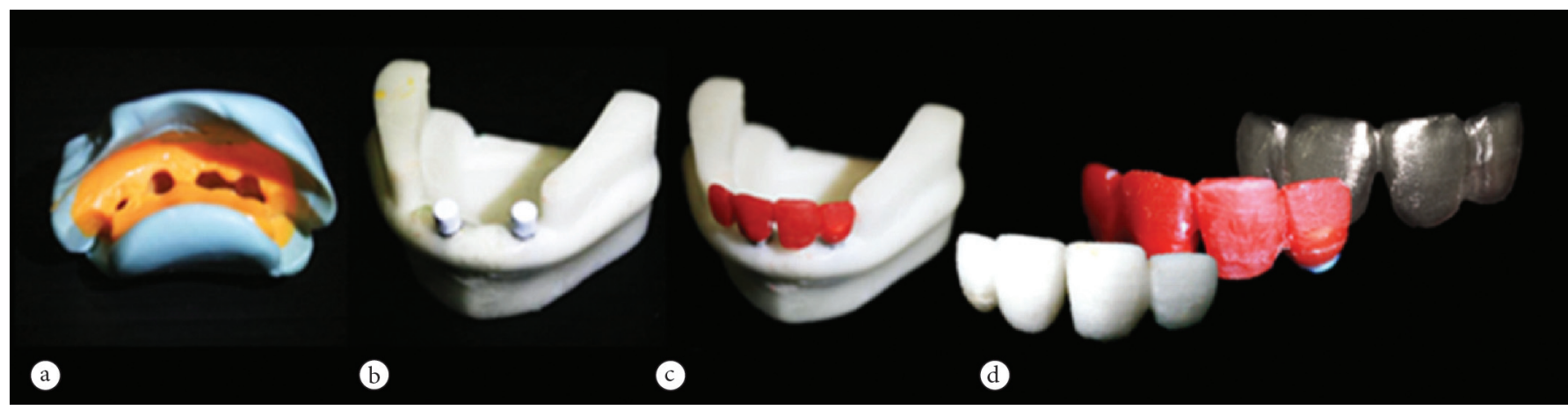

Figura 6. a-Molde; b-Cilindros calcináveis em posição; c-Prótese em resina Duralay; d-Fundição em liga de CoCr.

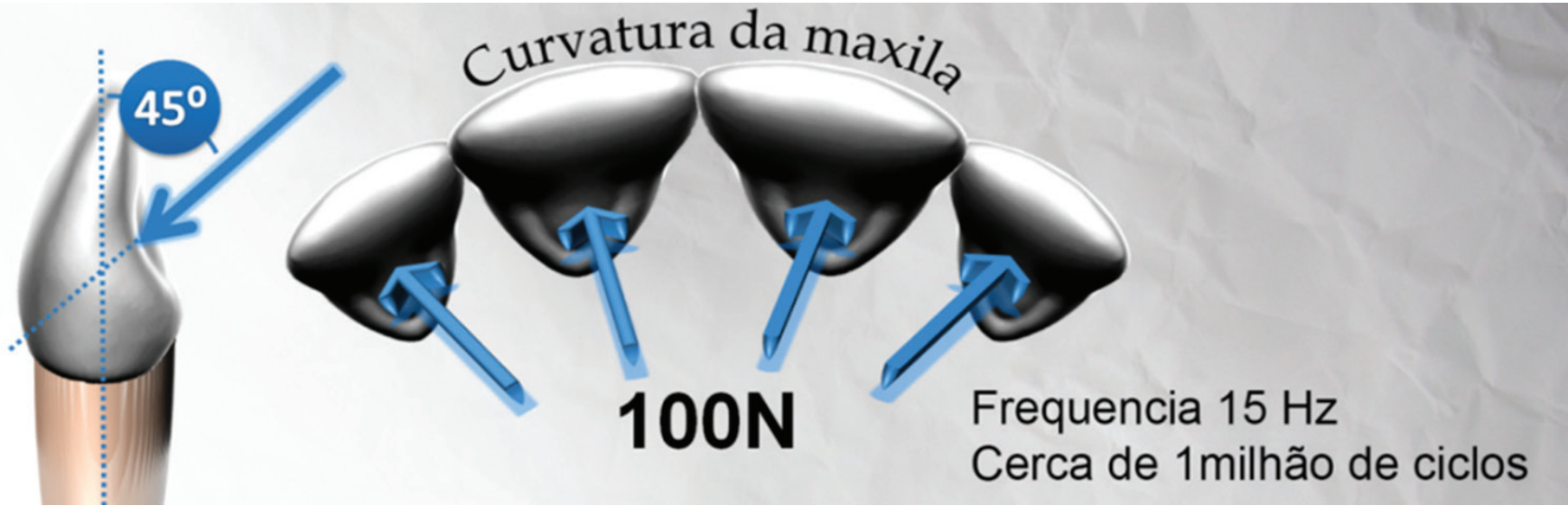

Figura 7. Direcionamento da aplicação de carga. 


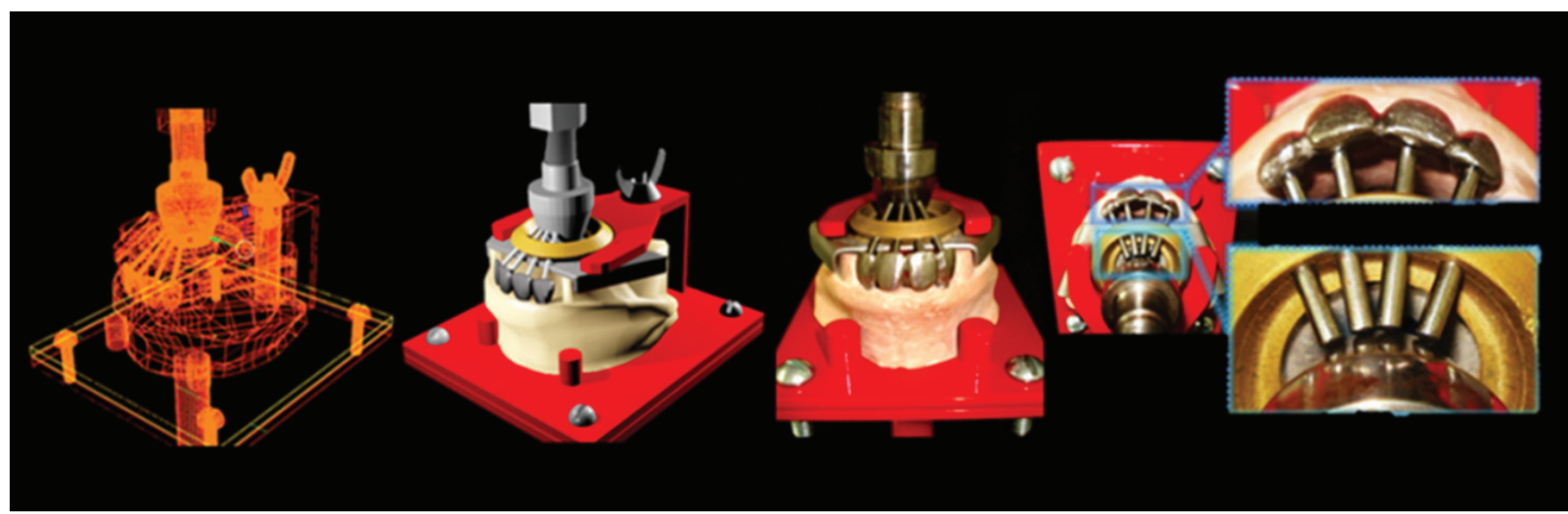

Figura 8. Dispositivo de aplicação de carga.

o carregamento simultâneo no cíngulo dos dentes anteriores superiores (Figura 8).

Para a simulação da função mastigatória na região anterior, uma força de $100 \mathrm{~N}$ e $15 \mathrm{~Hz}$ com uma angulação vertical de $45^{\circ}$ em relação ao longo eixo do dente foi aplicada no cíngulo de cada elemento dental ${ }^{16}$.

Os modelos foram submetidos ao ensaio de ciclagem mecânica, com um milhão de ciclos completos, o que representa cerca de 50 anos de função mastigatória ${ }^{14,18}$.

Para a ciclagem mecânica, foi utilizada a máquina de ensaios mecânicos MTS 810 (MTS System Corporation, Minneapolis, USA), equipada com o programa Gerador de Função do sistema FlexTest 40.

\section{RESULTADO}

A Tabela 1 representa os três grupos com as disposições de implantes estudados e a média dos seus respectivos números de ciclos realizados para cada conexão protética.

Após o ensaio de ciclagem mecânica, todos os modelos de todos os grupos com as três conexões protéticas atingiram cerca de um milhão de ciclos sem que ocorresse ruptura do parafuso do componente, do componente ou da estrutura metálica da prótese, ou qualquer tipo de falha visível a olho nu. Sabendo-se que 100 mil ciclos correspondem a um período de cinco anos de mastigação ${ }^{14,18}$, os resultados deste estudo equivalem a 50 anos de função mastigatória sem o aparecimento dos estágios de fadiga 2 e 3 , segundo Cetlin, Silva ${ }^{12}$.

\section{DISCUSSÃO}

Como descrito na literatura, encontra-se uma taxa de 90-95\% de sucesso no processo de osseointegração ao estabelecer o protocolo de Branemark. ${ }^{1}$ Porém, falhas ainda são observadas neste processo, as quais são classificadas entre falhas precoces ou $\operatorname{tardias}^{19}$.

As falhas precoces são as falhas que acontecem logo após a cirurgia e são caracterizadas pela falta de osseointegração. As falhas tardias acometem os implantes após a instalação da prótese. Existem duas explicações para as causas das falhas tardias
Tabela 1. Número de ciclos de ciclagem mecânico

\begin{tabular}{cccc}
\hline & Cone-Morse & $\begin{array}{c}\text { Hexágono } \\
\text { Externo }\end{array}$ & $\begin{array}{c}\text { Hexágono } \\
\text { Interno }\end{array}$ \\
\hline Grupo A & 1003231 & 1004656 & 1007021 \\
Grupo B & 1004071 & 1008256 & 1006413 \\
Grupo C & 1008538 & 1003711 & 1005807 \\
\hline
\end{tabular}

em implantes: 1) Perda de tecidos de suporte por infecções ou peri-implantites e 2) Problemas mecânicos, incluindo fraturas, sendo a sobrecarga biomecânica a mais frequente causa das falhas $\operatorname{tardias}^{19}$.

De acordo com McDermott et al. ${ }^{20}$, além da excessiva carga oclusal, outros fatores - como a localização do implante (maxila ou mandíbula, posteriores ou anteriores); o número insuficiente de implantes suportando uma prótese; o material com que a prótese é confeccionada, e o diâmetro do implante menor do que $3,5 \mathrm{~mm}$ - estão relacionados com a falha tardia do implante dentário.

Neste estudo, testando um carregamento funcional em uma reabilitação anterior de maxila por meio da ciclagem mecânica, foram obtidos excelentes resultados com relação ao comportamento mecânico dos implantes, componentes e parafusos protéticos, e da estrutura metálica. Ao observar que a colocação de implantes dentários só pode ser realizada na idade adulta, os resultados aqui obtidos demonstram uma excelente longevidade das estruturas utilizadas. Deste modo, os resultados deste estudo vêm acrescentar mais informações que possibilitam ao profissional reabilitar com maior segurança a região anterior de maxila.

\section{CONCLUSÃO}

Com a metodologia e as condições empregadas, pode-se concluir que, independentemente do posicionamento dos implantes e dos tipos de conexões protéticas utilizadas em uma reabilitação anterior de maxila, observa-se o mesmo comportamento mecânico. 


\section{REFERÊNCIAS}

1. Jemt T, Lekholm U, Adell R. Osseointegrated implants in treatment of patients with missing teeth--preliminary study of 876 implants. Quintessenz. 1990; 41: 1935-46.

2. Misch CE, Suzuki JB, Misch-Dietsh FM, Bidez MW. A positive correlation between occlusal trauma and peri-implant bone loss: literature support. Implant Dent. 2005; 14: 108-16. http://dx.doi.org/10.1097/01.id.0000165033.34294.db

3. Skalak R. Biomechanical considerations in osseointegrated prostheses. J Prosthet Dent. 1983; 49: 843-8. http://dx.doi.org/10.1016/00223913(83)90361-X

4. Baggi L, Cappelloni I, Di Girolama M. The influence of implant diameter and length on stress distribution of osseointegrated implants related to crestal bone geometry: a threedimensional finite element analysis. J Prosthet Dent. 2008; 100: 422-31. http://dx.doi.org/10.1016/ S0022-3913(08)60259-0

5. Barbier L, Schepers E. Adaptative bone remodeling around oral implants under axial and nonaxial loading conditions in the dog mandible. Int J Oral Maxillofac Implants. 1997; 12: 215-23.

6. Askary AS. Multifaceted aspects of implant esthetics: the anterior maxilla. Implant Dent. 2001; 10: 182-91. http://dx.doi. org/10.1097/00008505-200107000-00008

7. Choquet V, Hermans M, Adriaenssens P, Daelemans P, Tarnow DP, Malevez C. Clinical and radiographic evaluation of the papilla level adjacent to single-tooth dental implants: a retrospective study in the maxillary anterior region. J Periodontol. 2001; 72: 1364-71. http:// dx.doi.org/10.1902/jop.2001.72.10.1364

8. Tarnow DP, Cho SC, Wallace SS. The effect of inter-implant distance on the height of inter-implant bone crest. J Periodontol. 2000; 71: 5469. http://dx.doi.org/10.1902/jop.2000.71.4.546

9. Salama H, Salama MA, Garber D, Adar P. The interproximal height of bone: a guidepost to predictable aesthetic strategies and soft tissue contours in anterior tooth replacement. Pract Periodontics Aesthet Dent Pract. 1998; 10: 1131-41.

10. Gastaldo JF, Cury PR, Sendyk WR. Effect of the vertical and horizontal distances between adjacent implants and between a tooth and a implant on the incidence of interproximal papilla. J Periodontol. 2004; 75: 1242-6. http://dx.doi.org/10.1902/jop.2004.75.9.1242

11. Grunder U, Gracis S, Capelli M. Influence of 3-D bone to implant relationship on esthetic. Int J Periodontics Restorative Dent. 2005; 25 : 113-9.

12. Cetlin PR, Silva PSP. Análise de fraturas. São Paulo: Associação Brasileira de Metais; 1988.

13. Hoyer SA, Stanford CM, Buranadham S, Fridrich T, Wagner J, Gratton D. Dynamic fatigue properties of the dental implant-abutment interface: joint opening in wide-diameter versus standard-diameter hex-type implants. J Prosthet Dent. 2001; 85: 599-607. http://dx.doi. org/10.1067/mpr.2001.115250

14. Merz BR, Hunenbart S, Belser UC. Mechanics of the implant-abutment connection: an 8 degree taper compared to a butt joint connection. Int J Oral Maxillofac Implants. 2000; 15: 519-26.

15. Huang HM, Tsai CM, Chan CC, Lin CT, Lee SY. Evaluation of loading conditions on fatigue-failed implants by fracture surface analysis. Int J Oral Maxilofac Implants. 2005; 20: 854-9.

16. Tsuge T, Higawara Y. Influence of lateral-oblique cyclic loading on abutment screw loosening of internal and external hexagon implants. Dent Mater J. 2009; 28: 373-81. http://dx.doi.org/10.4012/dmj.28.373

17. Correa CB, Pires JR, Fernandes-Filho RB, Sartori R, Vaz LG. Fatigue and fluoride corrosion on Streptococcus mutans adherence to titanium-based implant/component surfaces. J Prosthodont. 2009; 18: 382-7. http://dx.doi.org/10.1111/j.1532-849X.2009.00463.x

18. Basten CHJ, Nicholls JI, Daly CH, Taggart R. Load fatigue performance of two implant-abutment combinations. Int J Oral Maxillofac Implants. 1996; 11: 522-8.

19. Piattelli A, Scarano A, Piattelli M, Vaia E, Matarasso S. Hollow implants retrieved for fracture: a light and scanning electron microscope analysis of 4 cases. J Periodontol. 1998; 69: 185-9. http://dx.doi.org/10.1902/jop.1998.69.2.185

20. McDermott NE, Chuang SK, Woo VV, Dodson TB. Complications of dental implants: identification, frequency, and associated risk factors. Int J Oral Maxillofac Implants. 2003; 18: 848-55.

\section{CONFLITOS DE INTERESSE}

Os autores declaram não haver conflitos de interesse.

\section{AUTOR PARA CORRESPONDÊNCIA}

Luis Geraldo Vaz

Departamento de Materiais Odontológicos e Prótese, Faculdade de Odontologia, UNESP - Univ Estadual Paulista, Rua Humaitá, 1680, 14800-000 Araraquara - SP, Brasil

e-mail: lugervaz@foar.unesp.br

Recebido: Julho 19, 2013

Aprovado: Novembro 1, 2013 